\title{
EL LÉXICO EN EL TRASTORNO ESPECÍFICO DEL LENGUAJE. PRINCIPIOS DE INTERVENCIÓN DESDE EL AULA
}

THE LEXICON IN SPECIFIC LANGUAGE DISORDER. PRINCIPLES OF INTERVENTION FROM THE CLASSROOM

Autor Ana Belén Martínez Lietos ${ }^{1}$,

Fecha de envío: 21 de mayo de 2020

Fecha de aceptación: 7 de junio de 2020

1 Psicóloga y Logopeda, Consejería de Educación y Deporte, de la Junta de Andalucia, anabelen_lietos@hotmail.com. 


\section{Resumen}

Alguna vez nos hemos planteado: ¿cómo aprendemos una nueva palabra?, ¿qué necesitamos? El aprendizaje del léxico se produce de forma incidental, en cualquier situación de la vida y en cualquier momento, pero también de un modo explícito, sobre el que vamos a vertebrar las pautas más adecuadas en este artículo. En los alumnos con Trastorno Específico del Lenguaje, entendido como el déficit que presentan a nivel léxico origina dificultades en la interacción social, en la comprensión, en la vida académica... ya que hay un marco de interrelaciones mutuas entre semántica, pragmática, morfosintaxis... que sitúa en desventaja a estos alumnos, aspectos que se analizarán las relaciones desde el modelo neuropsicolingüístico de Chevrie Muller, 1996. Bajo las investigaciones basadas en la evidencia, se aunarán todas las investigaciones que nos permitan establecer una nueva palabra en el léxico, los niños necesitan analizar la información fonológica, semántica y sintáctica disponible, y todas ellas han de estar presentes; en especial, en el alumnado con Trastorno Específico del Lenguaje. Se detallan los principios de intervención, desde el aula ordinaria en colaboración con los especialistas en la atención a este alumnado, bajo un modelo conjunto de intervención denominado mi diccionario.

Palabras clave: neuropsicológico, TEL, fonológica, sintáctica y semántica.

\section{Abstract}

Have we ever thought about how do we learn a new word? What do we need? The learning of the lexicon occurs incidentally, in any situation of life and at any time, but also in an explicit way, on which we will vertebrate the most appropriate guidelines in this article In students with Specific Language Disorder, the deficit they present at the lexical level, cause difficulties in social interaction, in understanding, in academic life... because there is a framework of mutual inter-relations between semantics, pragmatics, morphosytaxis.... that disadvantage 
these students, aspects that will analyze the relationships from the neuropsycholinguistic model of Chevrie - Muller, 1996. Under evidence-based research, all research will be joined, allowing us to establish a new word in the lexicon, children need to analyze the available phonological, semantic and syntactic information, and all of them must be present, especially in

\section{EL LÉXICO}

El Trastorno Especifico del Lenguaje, se puede definir como una "alteración significativa en la adquisición y desarrollo del lenguaje, que no está justificada por ninguna causa física, neurológica, intelectual ni sensorial, en unas condiciones sociales adecuadas" (Aguado et al, 2015).

El léxico es una de las áreas con mayor dificultad para los alumnos con Trastorno Específico del Lenguaje (TEL), motivo por el que hemos de tener claros principios de intervención en el aula, espacio idóneo para su desarrollo.

En el presente artículo vamos a describir los principios que, desde el aula, han de regir una adecuada students with Specific Language Disorder. The principles of intervention are detailed, from the ordinary classroom in collaboration with the specialists in the care of this student, under a joint model of intervention called my dictionary.

\section{Keyword:}

Neuropsycholinguistic, $S L D$, phonological semantic and syntactic

intervención a nivel léxico, con estrategias basadas en la evidencia.

Entre los aspectos clave para acercarnos al concepto de léxico, hemos de tener en cuenta que:

- Hemos usado a lo largo de este artículo el concepto léxico, entendido como el conjunto de palabras que conforman una determinada modalidad lingüística, y por extensión, también se denomina así a los diccionarios que los recogen, siendo el diccionario el modelo de intervención en el que nos hemos basado. Por otro lado, en ocasiones, se puede intercambiar con el término de vocabulario, pero entendemos que este queda más 
circunscrito al conjunto de palabras de una lengua que una persona conoce o emplea.

- Las palabras son los bloques que conforman el lenguaje, pero estos bloques están interconectados, como estudiaremos en el siguiente apartado, desde un enfoque neuropsicológico.

- Una palabra contribuye al significado de una oración y al mismo tiempo el significado de una palabra contribuye al significado de una oración y del contexto en el que aparece (Mendoza, 2016).

- Las palabras son fundamentales en el proceso de comprensión.

- Son la unión entre la cadena fonológica u ortográfica y un referente.

- Produce una unidad de significado, que se puede comprender.

- Supone una unidad de significado compartida entre personas

Dada la importancia del vocabulario en el proceso de aprendizaje, es una prioridad establecer procedimientos eficaces para mejorarlo.
El aprendizaje de las palabras se produce de forma incidental y con una enseñanza explícita. A esta última nos vamos a referir con las propuestas concretas que se desarrollarán para su implementación en el aula.

\section{MODELO}

\section{NEUROPSICOLINGÜÍSTICO (MNPL) NEUROPSICOLÓGICO}

Hemos de partir de un modelo neuropsicolingüístico, que nos permite estructurar los distintos procesos y funciones del lenguaje. Como puede verse en la figura 1 , el modelo MNLP (Chevrie-Muller, 1996, citado en Narbona y Chevrie-Muller, 2001), está organizado "horizontalmente" según dos vertientes: receptiva por un lado y expresiva, por otro.

Verticalmente, se detallan los distintos procesos implicados, organizados en tres niveles:

- Primario o sensoriomotor, desde la periferia hasta las áreas de procesamiento cortical.

- Secundario, de organización gnósica y práctica.

- Terciario, de operaciones psicolingüísticas, que incluye el conjunto de los procesos que intervienen en la comprensión y la expresión (fonología, léxico, 
morfosintaxis, semántica y pragmática) Siendo este tercer nivel indispensable en el modelo.

Es necesario delimitar claramente las relaciones y la ubicación que la semántica ocupa en el mismo, representada en rojo (Figura 1), las interrelaciones que aparecen detalladas entre:

- Semántica y pragmática, interdependencia mutua.
- Semántica, pragmática y comprensión, interdependientes.

- Semántica y morfosintaxis, interdependencia mutua.

- El reconocimiento fonológico, que condiciona la identificación léxica.

- La semántica condiciona la programación sintáctica.

- La selección léxica condiciona la programación fonológica... etcétera
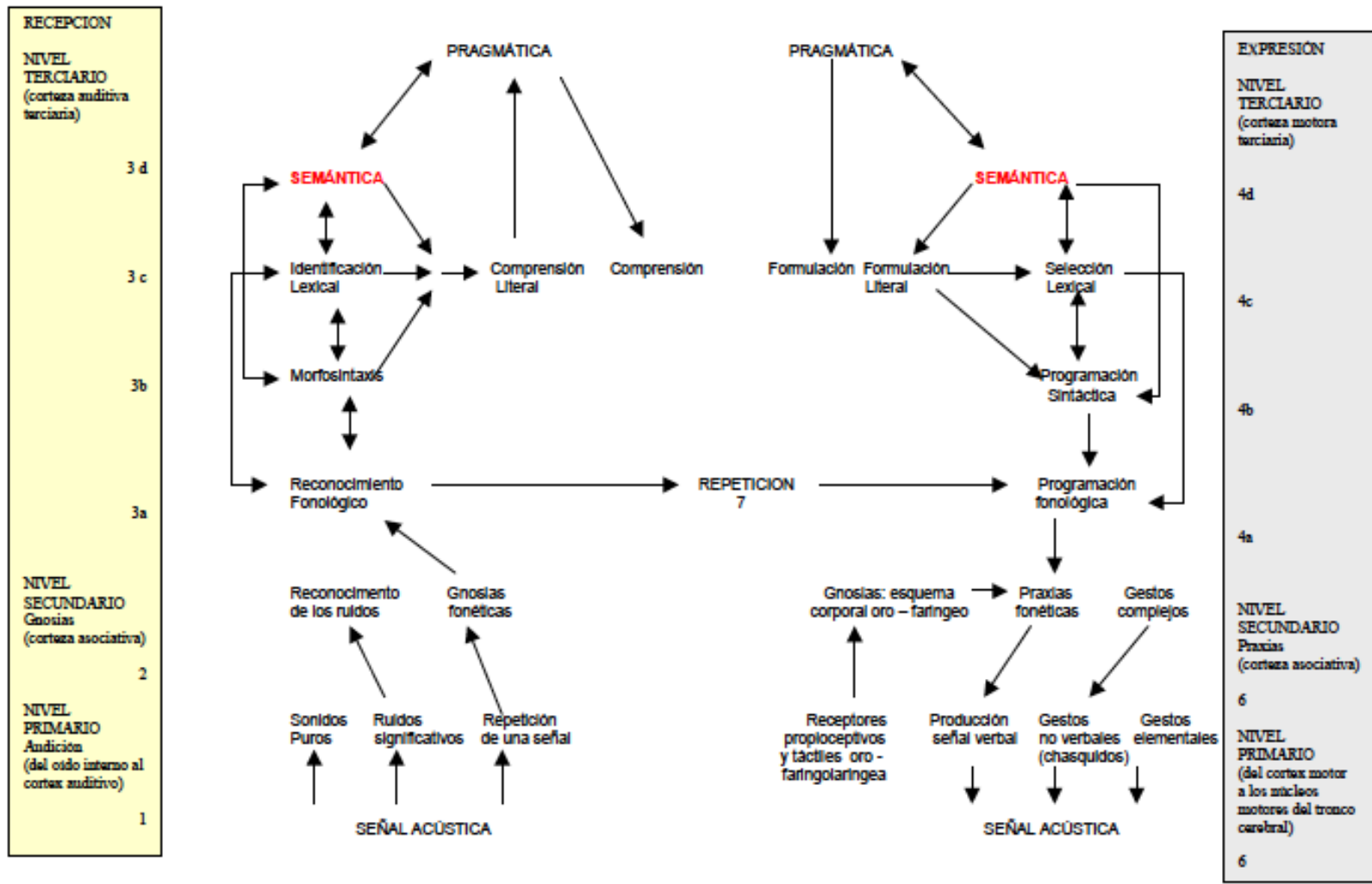

Figura 1: Modelo Neuropsicolingüístico (Chevrie-Muller, 1996, citado en Narbona y Chevrie- Muller y, 2001, p. 76)

Para un niño con TEL, el aprendizaje del vocabulario no es una tarea fácil. Tomando como referente el modelo MNPL anterior, los niños con TEL tendrían afectados los procesos a nivel terciario, marcados en rojo 
(Figura 2), pudiéndose originar un TEL léxico sintáctico, o fonológico sintáctico (Martínez, 2015)

Entre otras, las siguientes dificultades en relación con el léxico, atendiendo a los procesos detallados en este modelo son:

1- Presentan dificultades en el procesamiento del input, especialmente en cuanto al reconocimiento de palabras menos frecuentes (Dollaghan y Campbell, 1998, Coady, Evans y Kluender, 2010).

2- Bajo rendimiento en tareas de definición sugiere que sus representaciones semánticas están menos especificadas y precisas (McGregor y Appel, 2002).

3- Alteración en el procesamiento de las características fonéticas de las formas de las palabras (Montgomery y Evans, 2009).
4- Déficit en la memoria fonológica a corto plazo (Baddeley, 1990).

5- Mayor dificultad en añadir palabras nuevas al léxico y una menor eficiencia para acceder a palabras que conocen (Coady et al., 2010).

6- No utilizan adecuadamente el bootstrapping sintáctico para aprender nuevas palabras si no existen indicadores

semánticos o pragmáticos que le faciliten el aprendizaje.

$\mathrm{Si}$ la estructura gramatical es fácil, pueden extraer sin dificultad; no así con estructuras menos familiares:

1. El padre conduce el coche: sin dificultad

2. Al coche lo conduce el padre: difícil

3. El coche es conducido por el padre: difícil 

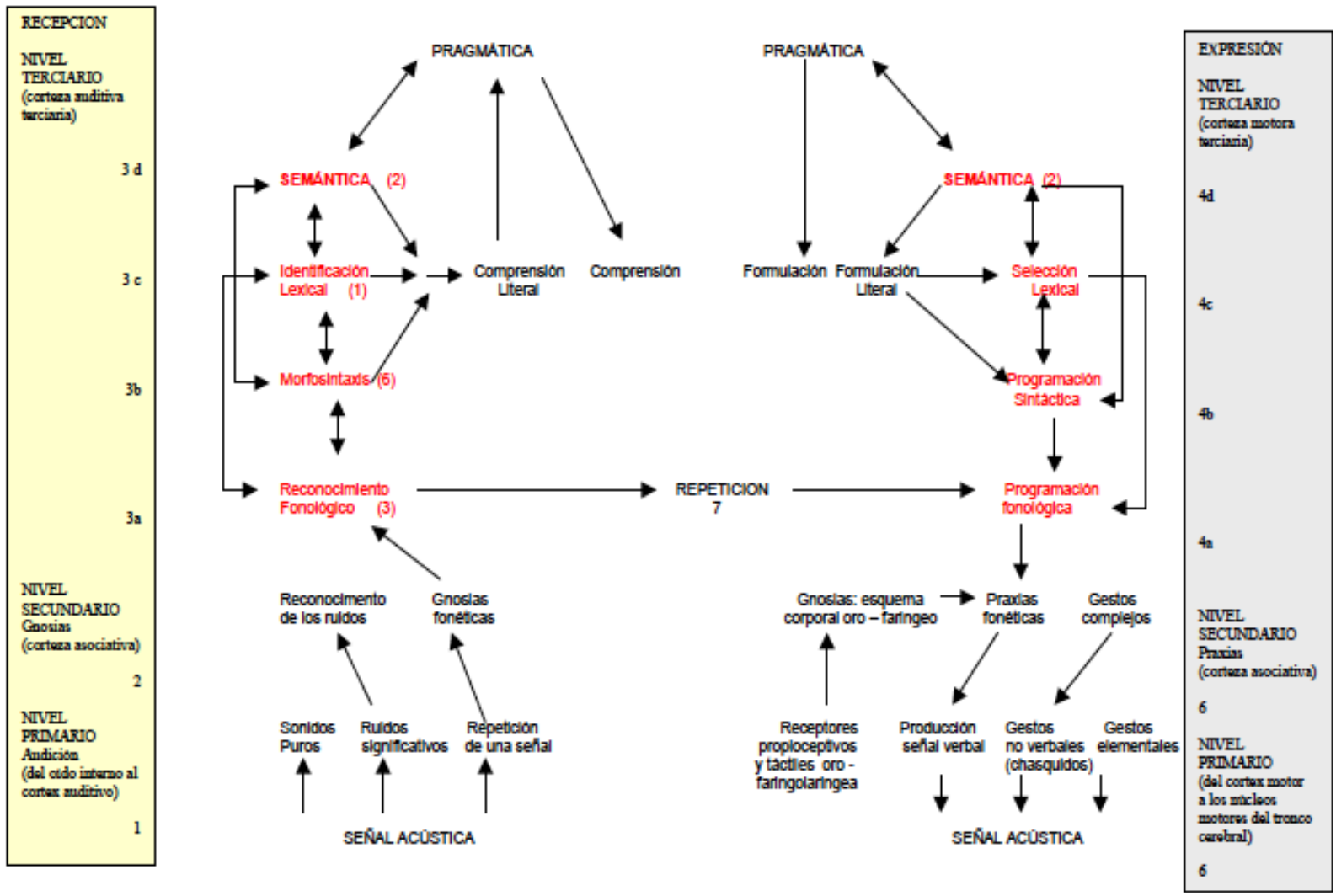

Figura 2. Alteraciones en el alumnado TEL, según MNPL (Chevrie-Muller, 1996, en Narbona y Chevrie- Muller, p. 76) representaciones léxicas

Los niños con TEL tienen dificultades de aprendizaje de palabras, tanto al principio, de forma incidental, como, posteriormente, con enseñanza explícita.

Diversas hipótesis estudian este proceso:

- Hipótesis del "almacenamiento": los niños no han aprendido las palabras de forma adecuada, por lo que son menos precisos y más lentos en la denominación (McGregor y Appel, 2002).

- Hipótesis del acceso para la recuperación: almacenadas son adecuadas, pero las palabras son menos accesibles.

Además, para evocar una palabra, es necesario disponer de:

- Información léxico-semántica.

- Información fonológica.

Estas dificultades, situadas en nuestro alumnado con TEL, originan que su léxico aparezca como la figura 3 , referida al verbo "nadar" en un niño con TEL. Ante esta situación, la evocación correcta del término se plantea muy complejo. 


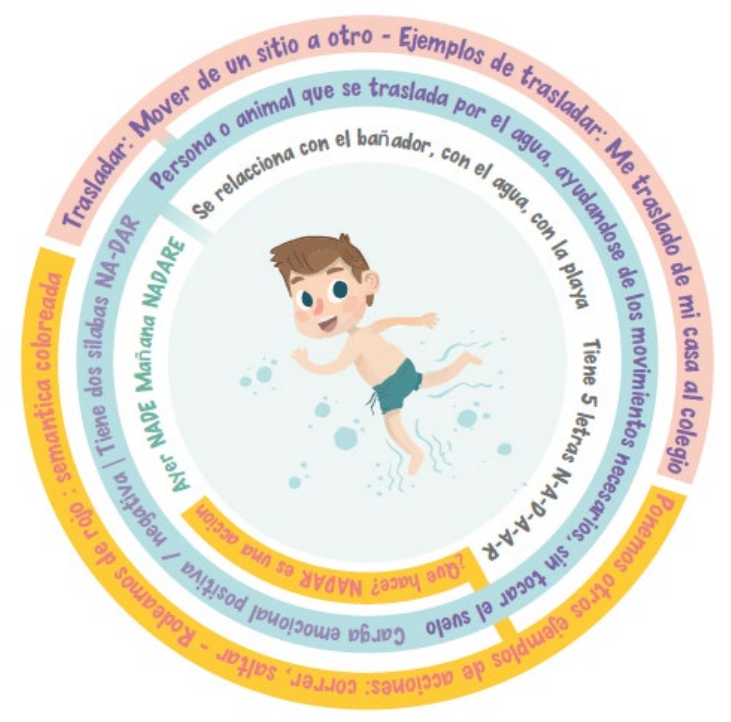

Figura 3. ¿Cómo aprende una nueva palabra un niño con TEL?

\section{EL APRENDIZAJE DESDE EL AULA}

En los centros educativos la variabilidad individual es la norma y no la excepción. Cuando los currículos son diseñados para atender la media imaginaria, no se tiene en cuenta la variabilidad real entre los estudiantes.

Estos currículos fracasan en el intento de proporcionar a todos los estudiantes unas oportunidades justas y equitativas para aprender, ya que excluyen a aquellos con distintas capacidades, conocimientos previos y motivaciones, que no se corresponden con el criterio ilusorio de "promedio", es necesario promover una inclusión educativa real, partiendo del "Index for Inclusion" de Booth, Ainscow, y Kingston (2006).
Es necesario intervenir en el léxico de los alumnos bajo una premisa conjunta, partir del nivel de desarrollo del alumno y ofrecer un modelo que permita ajustar la respuesta educativa. Steele y Mills (2011) apostaron por un modelo de integración del vocabulario en el ámbito escolar, prestando especial atención a diferentes estrategias de intervención del vocabulario curricular y enfatizando la intervención directa. Vamos a utilizar como ejemplo el verbo "nadar", pero se utilizaría en formato anticipación para todo el léxico a introducir a nivel curricular.

Principios básicos a desarrollar:

1. ¿Cómo se deben presentar las palabras? (Weismer y Hesketh, 1993):

- A ritmo lento, tanto para la producción como para la comprensión. En ocasiones presuponemos la comprensión y el ritmo es demasiado rápido.

- Énfasis en la entonación para la producción, ya que ayuda para la comprensión.

- Ayuda gestual para la comprensión, utilizando 
diversos formatos, desde gestos, pictos, dibujos lineales...

- A lo que añadimos que necesariamente se ha de partir del nivel de conocimiento del alumno; solo partiendo de su zona de desarrollo próximo, término acuñado por Vygosky, se producirá un verdadero aprendizaje significativo en nuestros alumnos.

- Además, requiere: Input repetitivo.

Estructura sintáctica simple y conocida.

Contexto rico que le ayude a la extracción del significado.

2. Requisitos de buena práctica en intervención del vocabulario (Stahl y Fairbanks, 1986):

- Proporcionar información contextual, que ayude a la comprensión.

- Enseñar en profundidad el significado de las palabras, no solo hemos de trabajar la amplitud, simultáneamente hemos de trabajar la profundidad del léxico.
- Proporcionar múltiples repeticiones y exposiciones a las nuevas palabras.

3. Lo que no se debe hacer (Graves 2006):

- Presentar la palabra fuera de contexto y pedir a los niños que la busquen en el diccionario.

- Hacer pruebas rápidas de palabras individuales.

- Completar laberintos de palabras (p. ej. crucigramas).

- Enseñar escritura más que vocabulario.

- Asumir que los indicadores contextuales son suficientes para adquirir el significado preciso de una palabra.

Para establecer una nueva palabra en el léxico, los niños necesitan analizar la información fonológica, semántica y sintáctica disponible, y todas ellas están presentes en el modelo de intervención que proponemos.

Messer y Dockrell (2013) sugieren que los programas de intervención en semántica son más efectivos, para 
favorecer la recuperación, que los de tinte fonológico. También se dispone de evidencia sobre la importancia de conjugar ambos.

El formato de intervención propuesta parte de una colaboración de todos los sectores de la comunidad educativa, donde cada uno ha de desarrollar su función específica y, sin un trabajo en equipo, esto no se va a lograr.

El enfoque a desarrollar en el aula enlaza con los principios básicos a tener en cuenta en el Programa Específico de los especialistas en la atención a la diversidad (que se desarrollará más adelante). Dependiendo del país en el que se desarrolle contaremos con maestros/as de audición y lenguaje, maestros las de pedagogía terapéutica, logopedas, fonoaudiólogos...

Fases a desarrollar en el aula ordinaria:

1. Brainstorming sobre la palabra en cuestión a desarrollar, por ejemplo, la palabra "nadar": ¿Qué sabemos de la palabra "nadar"? Es muy importante en esta fase, no coartar la capacidad comunicativa y/o creativa de nuestro alumnado, en esta fase "todo vale". Será el docente, el que desarrollará estrategias para reconducir las respuestas de los alumnos hacia la temática en cuestión.

\section{Introducción del significado} de las palabras. Debe iniciarse con definiciones claras y comprensibles por los niños, en lugar de guiarse por definiciones del diccionario, que no parece ser una forma efectiva para el aprendizaje de palabras. De hecho, es probable incluso, que los niños con desarrollo típico interpreten mal el significado (McKeown, 1993).

Es necesario proporcionar definiciones comprensibles por los niños, no definiciones de diccionario, ya que son más efectivas (Beck et al, 2008).

Mensualmente el Equipo Docente, entendida esta estructura como todos los docentes que imparten clase a un alumno, se reunirá con los especialistas en Atención a la Diversidad que intervienen con el alumnado (antes mencionados), para seleccionar el vocabulario sobre el que se va a trabajar, y la anticipación desde el Programa Específico. La selección del vocabulario partirá de todas las áreas de desarrollo del 
alumnado, con especial incidencia desde:

Lengua y Literatura.

Ciencias Naturales y Sociales. Matemáticas.

\section{Conciencia sintáctica. La} intervención sintáctica consistirá en la identificación de la categoría gramatical a la que pertenece la palabra trabajada, en base al uso de indicadores visuales. Vamos a utilizar los colores otorgados por Gloria López Garzón (1999) en el Programa "Enséñame a Hablar".

Con cada nuevo término introducido se responderá a las preguntas: ¿Quién?, ¿qué hace?, ¿cómo? y ¿qué es?; asignado un color a cada categoría gramatical: a) rojo $=$ verbos, $b)$ verde $=$ sujetos, $\mathrm{c}$ ) azul $=$ artículos y d) amarillo = sustantivos, adjetivos... (Figura 4).

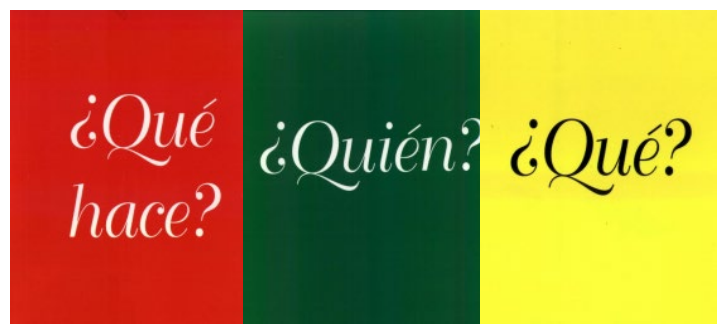

Figura 4. Código color enséñame a hablar: verbos, sujetos, objetos

Dentro del Plan de Atención a la Diversidad del centro se diseñarán actuaciones para la mejora de la competencia lingüística, que irán desde la prevención, detección, identificación e intervención con todo el alumnado en base a programas de intervención en semántica y fonológicos.

\section{Organizadores visuales. Al} finalizar la clase y para enlazar con la siguiente temática, realizaremos Organizadores visuales. Son los más adecuados para enseñar conceptos complejos (Graves, 2006) y se deben emplear para apoyar la definición y las actividades contextuales.

Entre los organizadores visuales más utilizados y eficaces a nivel léxico semántico se encuentran:

1. Los mapas semánticos

2. El análisis de las características semánticas

3. El diagrama de Venn.

Ejemplos con el tema que nos ocupa, el verbo "nadar" (figuras $5,6$ y 7$)$ : 


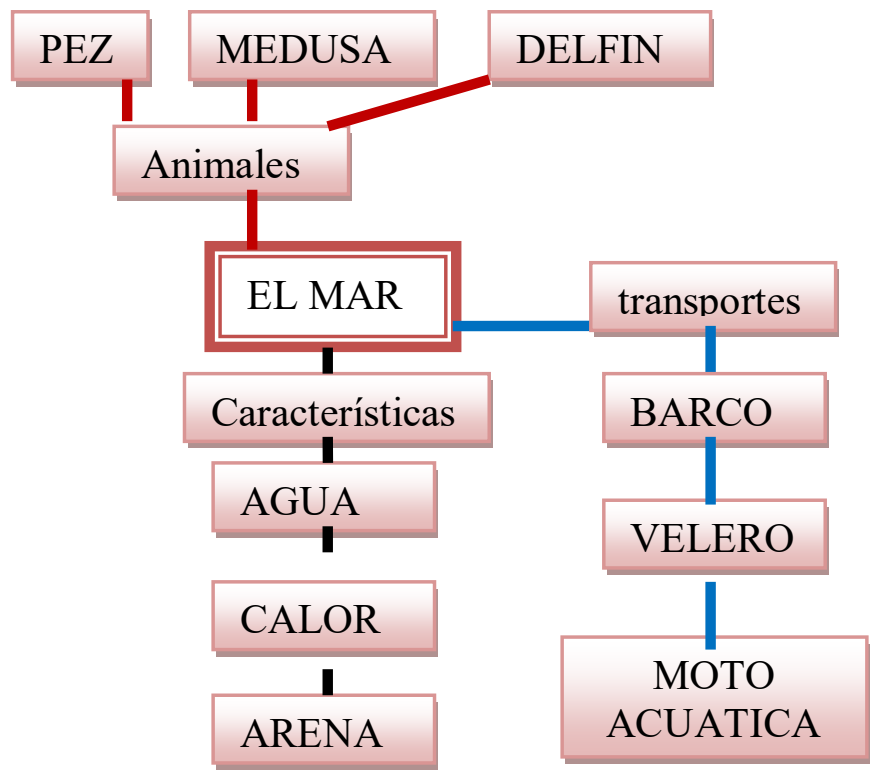

Figura 5. Mapa semántico el mar

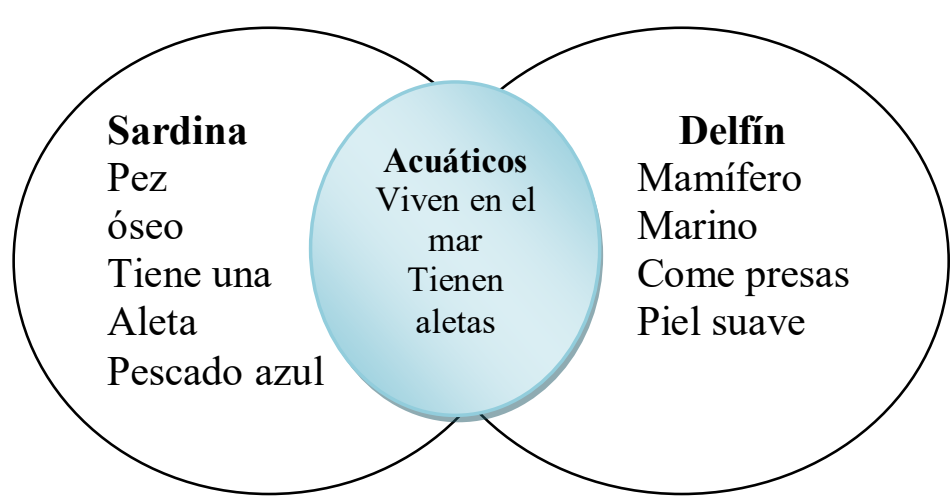

Figura 6. Diagrama de Venn con animales acuáticos

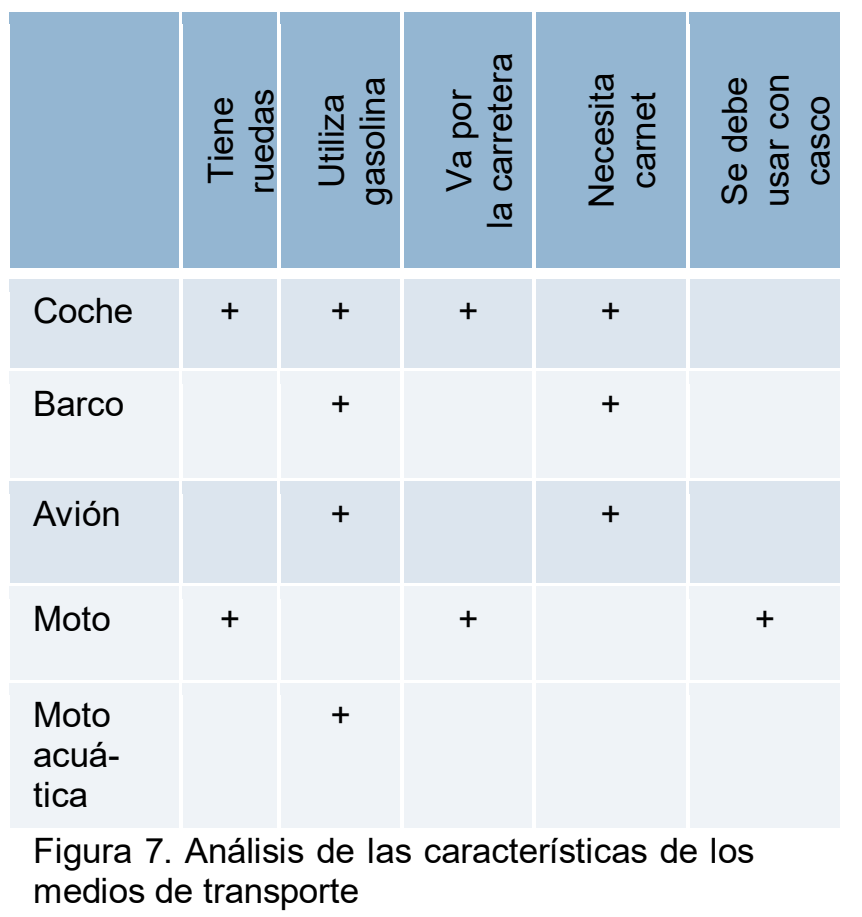

\section{INTERVENCIÓN CON LOS ESPECIALISTAS}

Todas las estrategias que nos marca la evidencia científica las hemos aunado en un solo documento denominado MI DICCIONARIO, con las siguientes fases a desarrollar:

Fase 1: Brainstorming sobre la palabra en cuestión a desarrollar, por ejemplo, la palabra nadar: ¿Qué sabemos de la palabra nadar? Exactamente igual que hemos realizado en el aula ordinaria, en este caso el especialista irá propiciando la competencia lingüística de los alumnos y que expresen sus opiniones al respecto, aunque no lo digan con un nivel de inteligibilidad adecuado.

De forma simultánea al aula ordinaria, los especialistas trabajarán el léxico.

Comenzamos con el aprendizaje de verbos, ya que los niños/as con TEL tienen más dificultades con el aprendizaje de verbos, por lo que la proporción de verbos en el léxico de los niños con TEL es inferior al esperado (Rice y Bode, 1993).

Tiene una mayor presencia de verbos de alta frecuencia. 
Estas diferencias se pueden deber:

- A la mayor complejidad de los verbos.

- Al mayor rol gramatical.

\begin{tabular}{|c|c|}
\hline NADAR & ¿Qué significa? \\
\hline & $\begin{array}{l}\text { Definición de la RAE. } \\
\text { Dicho de una persona o de un animal: Trasladarse en el agua, } \\
\text { ayudándose de los movimientos necesarios, y sin tocar el suelo ni } \\
\text { otro apoyo. } \\
\text { Esta definición no es adecuada para la mayoría de los alumnos de } \\
\text { nuestras aulas. Ejemplos de definiciones dadas por niños: } \\
\text { Carlos, } 9 \text { años: tirarse a la piscina y mover los brazos. }\end{array}$ \\
\hline $\begin{array}{l}\text { Número de sílabas } \\
\text { (cruces) } \\
\text { Conciencia silábica }\end{array}$ & $\begin{array}{l}\text { Julia, } 9 \text { años: mover los brazos en el agua. } \\
\text { Ambos coinciden en la necesidad del movimiento de los brazos; }\end{array}$ \\
\hline \begin{tabular}{l|l}
$\mathbf{X}$ & $\mathbf{X}$ \\
\end{tabular} & $\begin{array}{l}\text { ninguno habla de apoyo necesario, ni de trasladarse. Son términos } \\
\text { compleios y "poco necesarios" en las definiciones dadas por ellos. }\end{array}$ \\
\hline $\begin{array}{l}\text { Número de letras } \\
\text { (círculos) } \\
\text { Conciencia fonémica }\end{array}$ & $\begin{array}{l}\text { Justo de estas definiciones partirá nuestra intervención. } \\
\text { Necesariamente ha de partir de donde el alumno está en ese } \\
\text { momento y expandir e introducir más términos, poco a poco. }\end{array}$ \\
\hline 00000 & \\
\hline
\end{tabular}

Figura 8. Significado de las palabras

niños, no definiciones de diccionario,

Fase 2: Desarrollo de habilidades metalingüísticas: conciencia silábica y conciencia fonémica. Si observamos que el alumnado presenta dificultades en la segmentación léxica, añadiremos un apartado a esta fase (se detalla esta fase en la Figura 9).

Fase 3: Introducción del significado de las palabras.

Es necesario proporcionar definiciones comprensibles por los
- A la mayor asociación de verbos con acciones. ya que son

más efectivas (Beck et al., 2008), de hecho, es probable incluso que los niños con desarrollo típico interpreten mal el significado (McKeown, 1993).

Esta tercera fase comienza escribiendo la palabra encima del dibujo; la escritura con mayúsculas y minúscula a juicio del docente. Seguidamente vamos a definir la palabra "nadar" (Figura 8). 


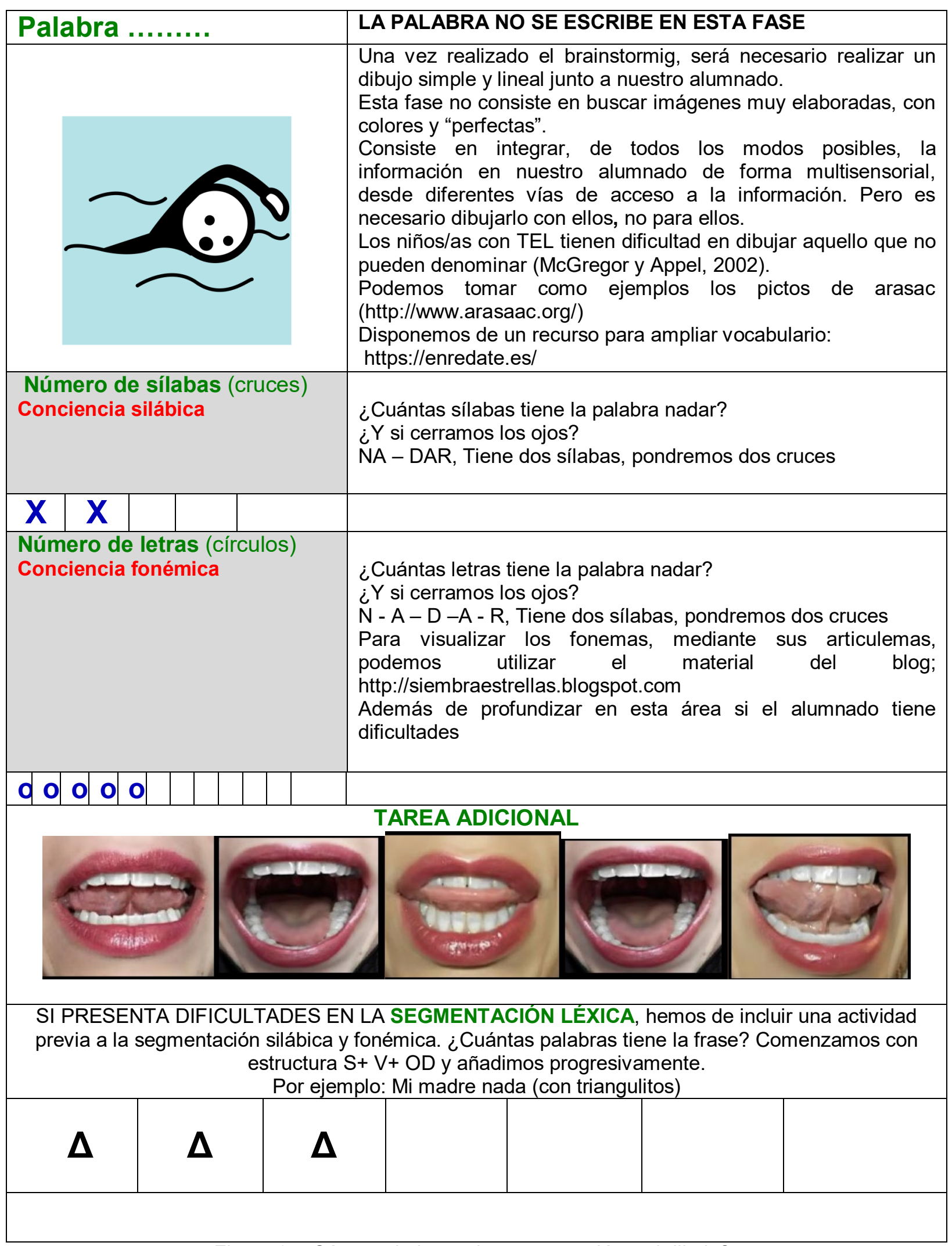

Figura 9. ¿Cómo trabajamos la segmentación y el dibujo? 
Fase 4: La conciencia morfológica.

Además, será necesario un entrenamiento en conciencia morfológica, entendida como la habilidad para reconocer, reflejar y manipular la estructura subléxica de las palabras.

Los niños incrementan de forma gradual su conciencia morfológica, influidos por las experiencias con palabras morfológicamente complejas mientras leen y escriben, así como por el grado de transparencia ortográfica, semántica y fonológica entre una palabra derivada o afijada y la palabra base.

La conciencia morfológica puede facilitar la conciencia fonológica en niños de edad escolar con trastornos de lenguaje, porque la instrucción con base morfológica incorpora la conciencia de sonidos o fonemas vinculando su información con el significado.

Ej.: Nadar, nadador, nadadora, nadando, natación.

La palabra-base "nad" sirve para averiguar el significado de los ejemplos anteriores, construyendo palabras derivadas y con diferentes sufijos que nos valen para diferenciar el significado de cada una.

La conciencia morfológica ayuda al incremento del vocabulario, los niños con dificultades lingüísticas tienen un vocabulario y unas formas morfológicas menos complejas que sus iguales, son menos precisos en el uso de esas formas y realizan las tareas morfológicas de forma similar a los niños más pequeños; por tanto, es probable que, sin instrucción explícita, los niños con dificultades de lenguaje no desarrollen un vocabulario productivo.

La conciencia morfológica facilita la comprensión lectora. A medida que los niños avanzan en su escolarización, se incrementa el número de palabras morfológicamente complejas. Ya que el significado de estas palabras se puede predecir por el de cada uno de sus componentes, una conciencia de la estructura morfológica de las palabras puede ayudar a los niños al aprendizaje de nuevas palabras que oyen o que leen.

La intervención se centrará en:

- Identificación de modelos morfológicos, a través del diccionario

- Enfoque explícito en las unidades morfológicas de significado, con estrategias de evocación en base a preguntas, trabajando conjuntamente:

Conciencia morfológica flexiva Conciencia morfológica derivativa 


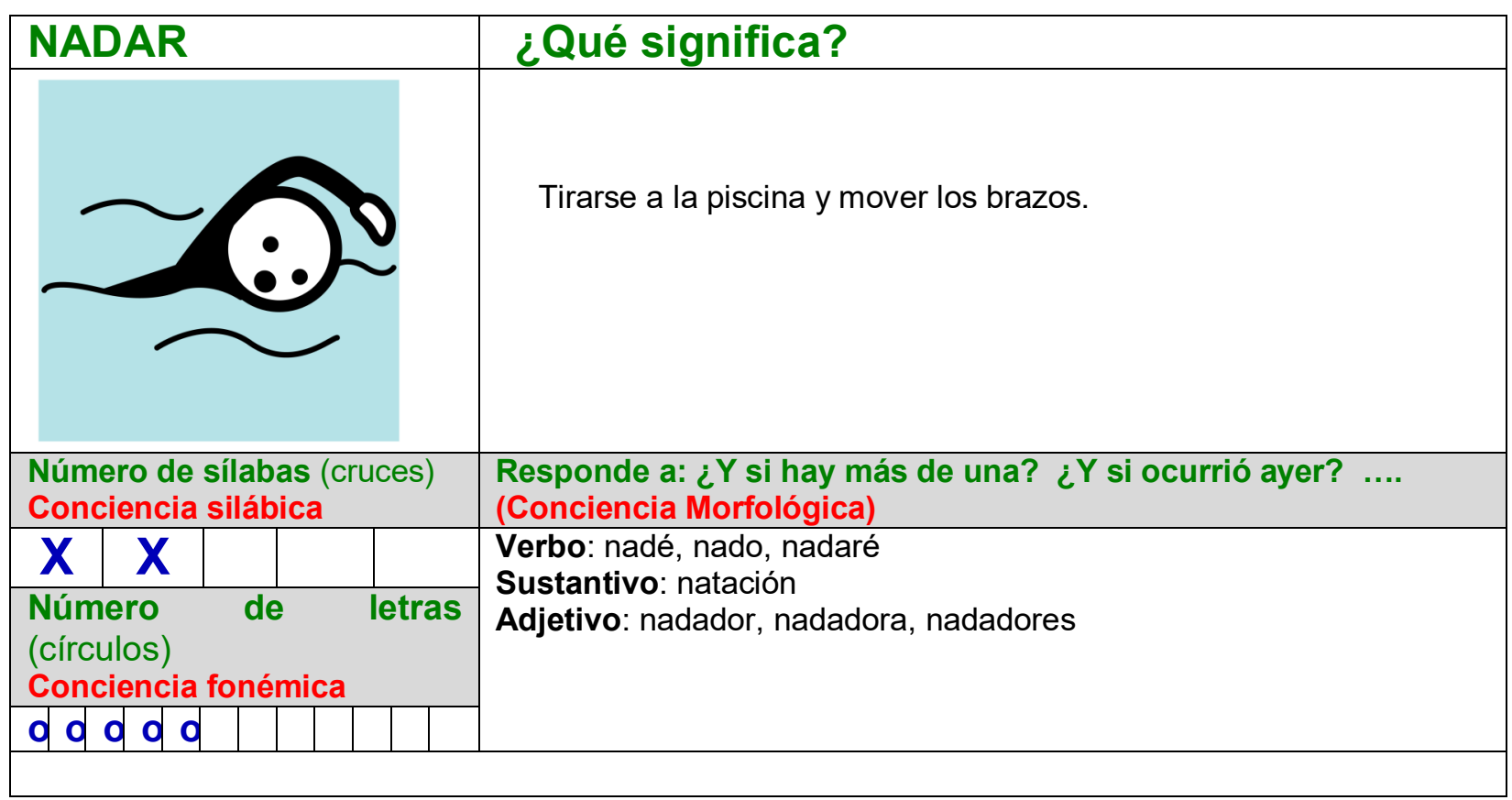

Figura 10. Conciencia morfológica

(nadador) ¿Una chica? (nadadora)

Es necesario tener en cuenta

¿Varias chicas? (nadadoras)

que dependiendo de la categoría gramatical de la palabra objeto de estudio, podrá tener unas (IMAGEN 10: CONCIENCIA posibilidades derivativas, flexivas $u$ otras.

En el caso que nos ocupa, al tratarse de un verbo, ofrece la siguiente información morfológica:

Es un verbo: por lo tanto, admite declinación verbal. En este caso evocaremos mediante preguntas del tipo ¿Y si lo hiciste ayer? (nadé) ¿Cómo lo dirías si lo vas a realizar mañana? (nadaré)

Sustantivo: natación

Adjetivo: nadador/a, género y número. Evocaremos con preguntas del tipo ¿Y si lo hace un chico? 
palabra trabajada, en base al uso de indicadores visuales.

Los indicadores visuales que se han descrito como más eficaces son: «Semántica coloreada»:

Códigos de color. Ejemplos:

SPS (Mayer-Johnson, 1981)

Enséñame a hablar (López
Fase 6: Incremento de la profundidad del significado de las palabras.

La riqueza de vocabulario de un individuo puede considerarse desde una doble perspectiva: la amplitud y la profundidad.

La amplitud se refiere al número de palabras que una persona

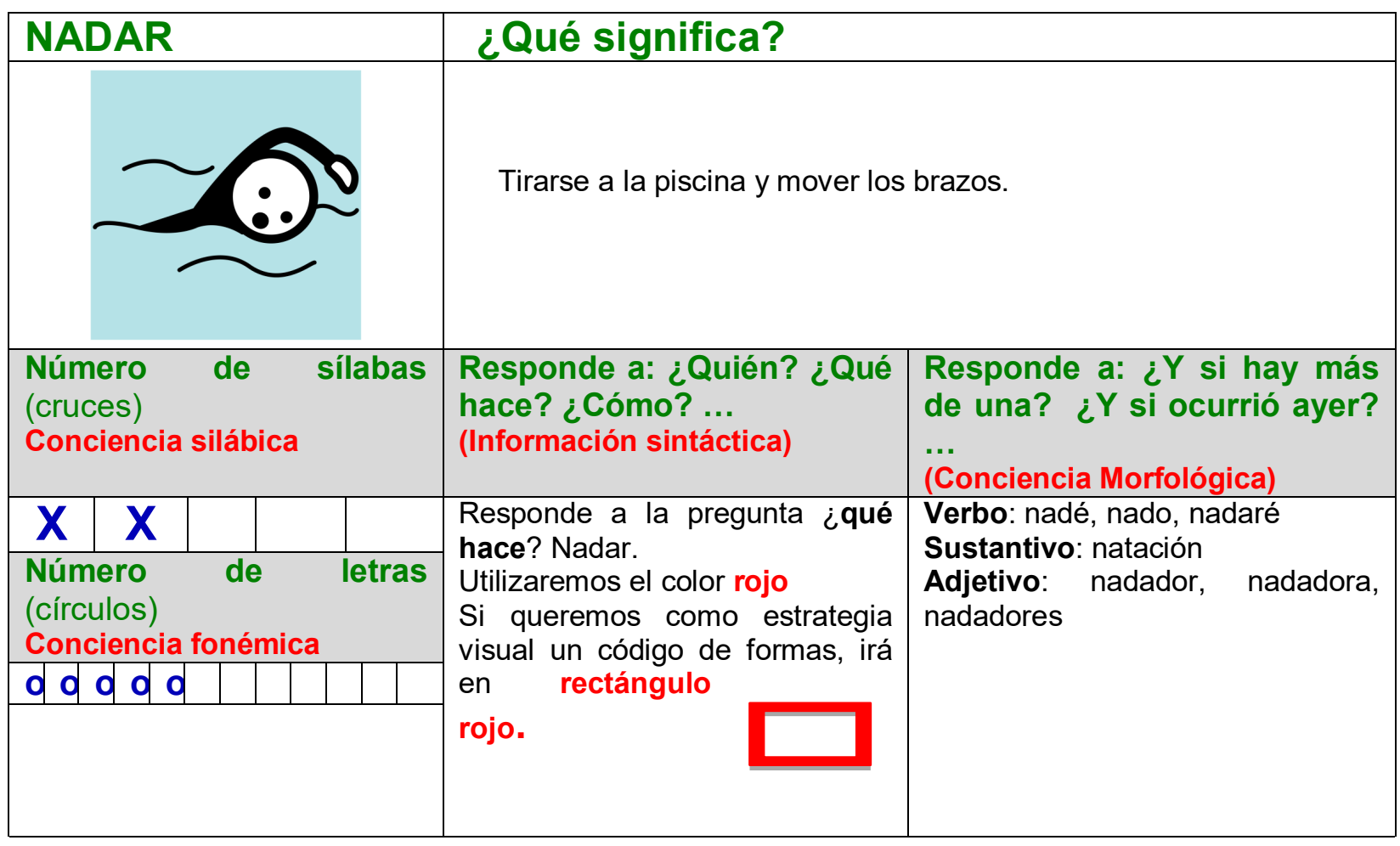

Garzón, 2013)

Puesto que en los centros educativos utilizan ampliamente el Figura 11: Información sintáctica

Enséñame a Hablar en las aulas de Audición y Lenguaje y Pedagogía Terapéutica, vamos a partir del código de color, usado por Gloria López Garzón. (Figura 11). conoce, pero es necesario ver hasta qué punto las conoce, es decir la profundidad (McGregor et al., 2013).

La amplitud, nos proporciona una comunicación precisa.

La profundidad, en cambio proporciona una flexibilidad, la comprensión sin apoyo contextual, una mejora en la calidad de las 
definiciones y permite relacionar palabras.

Ambos proporcionan un valor diferencial predictor en el deben discutir los contextos en los que rendimiento lector.

Diversas actividades ayudan a profundizar en el significado de las palabras, como búsqueda de sinónimos y antónimos, generación de ejemplos y no ejemplos de palabras, discusión sobre las semejanzas y diferencias entre una palabra nueva y otra conocida (Stahl y Nagy, 2006)

También pueden ser útiles las actividades de categorización y asociación.

Igual que en las fases anteriores, dependiendo de la palabra en sí, podremos responder a estas preguntas o a otras (Figura 12).

Fase 7: Expansión de las palabras a nuevos contextos.

Una vez trabajada la palabra, ésta se debe integrar en el contexto de una oración o de un párrafo completo puede aparecer.

Imagen 12: Incremento de la profundidad

Se realizarán entre otras las siguientes actividades:

Producir oraciones utilizando las palabras aprendidas (Graves, 2006)

Completar una oración que contiene la palabra-objeto

Generación de narraciones con las palabras-objeto

Generación de Organizadores visuales, como los mapas semánticos, análisis de las relaciones semánticas, y los diagramas de Venn (Figura 13).

Recomendamos para el desarrollo de actividades y como guía las obras de Gerardo Aguado, Juan Cruz Ripoll Salceda y María José Domezáin (2003), y la de Marc Monfort y Adoración Juárez (2004). y se

\section{Aprendemos más}

\begin{tabular}{|c|c|c|}
\hline ¿Para qué sirve? & ¿Con qué se relaciona? & ¿De qué color es? \\
\hline Para no ahogarte & $\begin{array}{l}\text { Asociaciones: agua- nadar, } \\
\text { bañador - nadar, nadar - mar, } \\
\text { piscina (evocación de términos) }\end{array}$ & \\
\hline Sinónimos & Antónimos & $\begin{array}{l}\text { Tiene tilde en... Se escribe con } \\
\text { v/b... } \\
\text { Representación ortográfica }\end{array}$ \\
\hline Flotar, bucear, bañarse & $\begin{array}{l}\text { AÑO 2020, VOLUMEN } 4 \\
\text { Hundirse, sumergirse }\end{array}$ & \begin{tabular}{c|c|} 
Nadar & 18 \\
Se escribe con r al final
\end{tabular} \\
\hline
\end{tabular}




\section{Escribimos frases con distintos significados}

Contexto lingüístico: Tú nadas muy bien sin flotador (nadas: segunda persona del singular del presente de indicativo del verbo nadar)

Contexto extralingüístico: Ayer mis padres decidieron comprar un sofá nuevo. Mi padre quería uno que costaba 1.500 euros y mi madre uno que tan solo costaba 800 euros. Mi madre enfadada, le dijo a mi padre: ¡Claro, como tú nadas en la abundancia

Imagen 13: Expansión de las palabras

\section{DICCIONARIO DE PALABRAS}

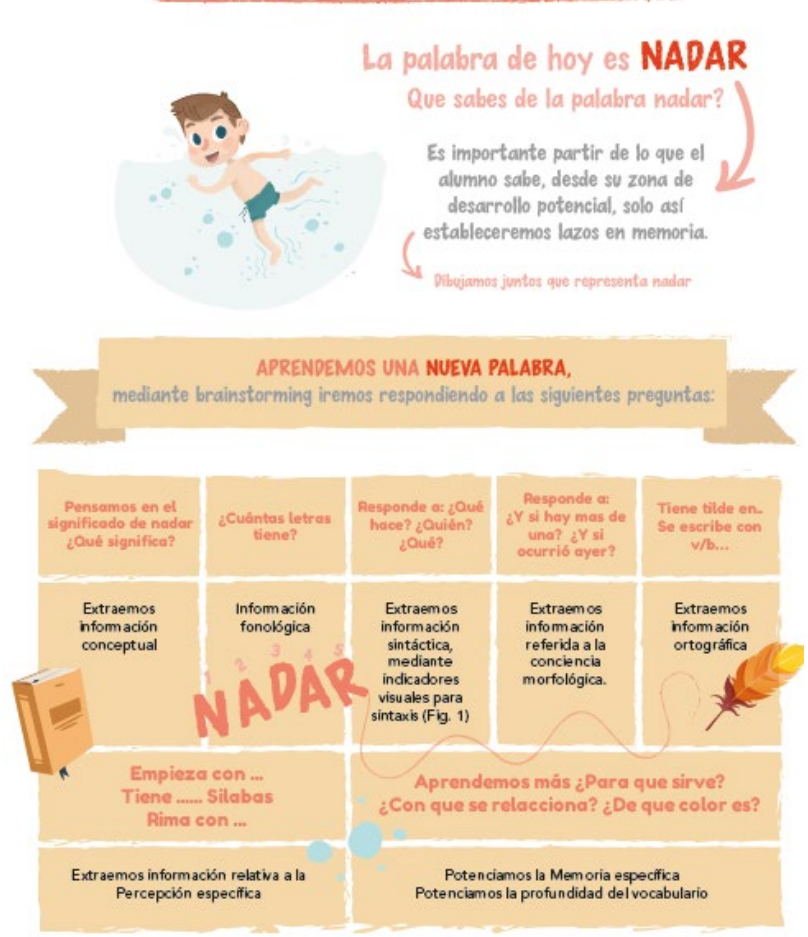

Figura 14. Mi DICCIONARIO

\section{CONCLUSIONES}

La intervención a nivel léxico bajo las premisas detalladas o solo beneficia

al alumnado con Trastorno especifico del Lenguaje, es una herramienta que beneficia a todo el alumnado, otorgando una verdadera educación inclusiva, desde nuestras aulas, con un trabajo cooperativo y colaborativo entre todos los que intervienen sobre el alumnado en cuestión, bajo el modelo de Mi Diccionario (figura 14) que hemos ido referenciando a lo largo de todo el documento.

La intervención desarrollada, a lo largo del presente curso escolar, ha permitido aunar una intervención específica basada en la evidencia, con un buen soporte teórico, con una intervención directa y extrapolable al aula ordinaria, lugar en el que verdaderamente ocurren los aprendizajes de calidad y la interacción social.

Aún nos queda presentar los resultados del diseño cuasi 
experimental que se está desarrollando en la actualidad con un grupo de niños con TEL, así como la comprobación a nivel del léxico en el grupo de iguales del mismo curso de referencia, en el que se encuentran escolarizados los alumnos objeto de estudio.

\section{REFERENCIAS BIBLIOGRÁFICAS}

Aguado, A., Coloma, C.J., Martínez Lietos, A.B., Mendoza, E., Montes, A., Navarro, R. y Serra, M. (2015). Documento de consenso elaborado por el comité de expertos en TEL sobre el diagnóstico del trastorno. Revista de Logopedia, Foniatría y Audiología, 35 (4), 147-149. http://dx.doi.org/10.1016/j.rlfa.2015.06. 004

Aguado, G., Juan Cruz Ripoll Salceda, J. C. y Domezáin, M. J. (2003). Comprender el Lenguaje haciendo ejercicios. Madrid: Entha.

Baddeley, A.D. (2000). The episodic buffer: A new component of working memory. Trends in Cognitive Sciences, 4, $417-423$

Beck, I., McKeown, L y Lucan, M (2008). Creating robust vocabulary:
Frequently asked questions and extended examples. Nueva York: The Guilford Press.

Booth, T., Ainscow, M. y Kingston, D. (2006). Index para la Inclusión. Desarrollo del juego, el aprendizaje y la participación en Educación Infantil. Bristol: CSIE.

Coady, J.A., Evans, J.L y Kluender, K.r. (2010). The role of phonotactic frequency in nonword repetition by children with specific language impairments. International Journal of Language and Comunication Disorders, 43, 1-40.

Dollaghan, C.A. y Campbell, T (1998). Nonword repetition and child language impairment. Journal of Speech, Language, and Hearing Research, 41, $1136-1146$

Fresneda, D., Mendoza, E., Muñoz, J., y Carballo, G. (2012). La práctica basada en la evidencia en la logopedia española: actitudes, usos y barreras. Revista de Investigación Educativa, 30 , 29-52.

doi: 10.6018/rie.30.1.111551 
Graves, M.F. (2006). The vocabulariy book: Learning and instrucción. Nueva York: Teacher College Press.

López Garzón, G. (1999). Programa "Enséñame a Hablar". Granada: Grupo Editorial Universitario.

Martínez Lietos, A. B. (2015). Identificación de dos perfiles de TEL con el CELF 4, WISC IV y el FON. Propósitos y representaciones: Revista de Psicología Educativa de laUSIL http://dx.doi.org/10.20511/ pyr2015.v3n2.78

McGregor, K.K. y Appel, A (2002). On the relation between mental representation and naming in a child with specific lenguaje impairment. Clinical LInguistics and Phonetics, 16, $1-20$

McGregor, K., Oleson, J. J., Bahnsen, A. y Durr, D, (2013). Children with developmental language impairmente have vocbulary déficits characterized by limited breadth and depth. Journal of Language and Communication Disorders, 48, 307-319

McKeown, M.G. (1993). Creating efective definitions for Young Word learners, Reading Research Quarterly, 28, 16-31

Mendoza, E. (2016). Trastorno especifico del lenguaje (TEL): Avances de un trastorno invisible. Madrid: Pirámide.

Messer, D., \& Dockrell, J. E. (2013). Children with word finding difficulties: Continuities and profiles of abilities. First Language, 33(5), 433-448. doi: https://doi.org/10.1177/014272371349 3345

Monfort, M. y Juárez, A. (2004). Leer para hablar: la adquisición del lenguaje escrito en niños con alteraciones del desarrollo y/o del lenguaje. Madrid: Entha.

Montgomery, J. W. y Evans, J.L. (2009). Complex sentence comprehension and working memory in children with specific language impairment. Journal of Speech, Language, and Hearing Reseach, 52, 269-288

Narbona, J y Crevrie -Muller, C (2001). El lenguaje del niño: desarrollo normal, evaluación y trastornos. Barcelona: Masson. 
Rice, M. L., \& Bode, J. V. (1993). GAPS in the verb lexicons of children with specific language impairment. First Language, 13 (37), 113-131. doi: https://doi.org/10.1177/014272379301 303707

Stahl, S.A. y Fairbanks, M.M. (1986) The effects of vocabulary instruction: $A$ model - based meta - analysis. Review of Educational Research, 56, $72-100$.

Steele, S. y Mills, M. T. (2011). Vocabulary intervention for school age children with language impairment: A review of evidence and good practice. Child Languaje Teacning and Therapy, 27, 354-370.

Weismer, S. E. y Hesketh, L. J. (1993). El impacto del estrés enfático en el aprendizaje de palabras nuevas por parte de niños con problemas específicos del lenguaje https://doi.org/10.1044/js/hr.4106.1 444

\section{Webgrafía}

http://www.arasaac.org/

https://enredate.es/

http://siembraestrellas.blogspot.com 